\title{
Transparent films made of highly scattering particles
}

Talha Erdem ${ }^{1,2, *}$, Lan Yang ${ }^{3}$, Peicheng Xu${ }^{1}$, Yemliha Altintas ${ }^{2}$, Thomas O’Neil ${ }^{1}$, Alessio Caciagli $^{1}$, Caterina Ducati ${ }^{4}$, Evren Mutlugun ${ }^{2}$, Oren A. Scherman ${ }^{3}$, Erika Eiser ${ }^{1, *}$ ${ }^{1}$ Cavendish Laboratory, Department of Physics, University of Cambridge, JJ Thomson Avenue, Cambridge CB3 OHE, United Kingdom

${ }^{2}$ Abdullah Gül University, Departments of Electrical-Electronics Engineering, and Materials Science and Nanotechnology Engineering, 38080, Kayseri, Turkey

${ }^{3}$ Melville Laboratory for Polymer Synthesis, Department of Chemistry, University of Cambridge, Lensfield Road, Cambridge CB2 1EW, United Kingdom ${ }^{4}$ Department of Material Science and Metallurgy, University of Cambridge, 27 Charles Babbage Road, Cambridge CB3 OFS, United Kingdom

\section{Supporting Information}
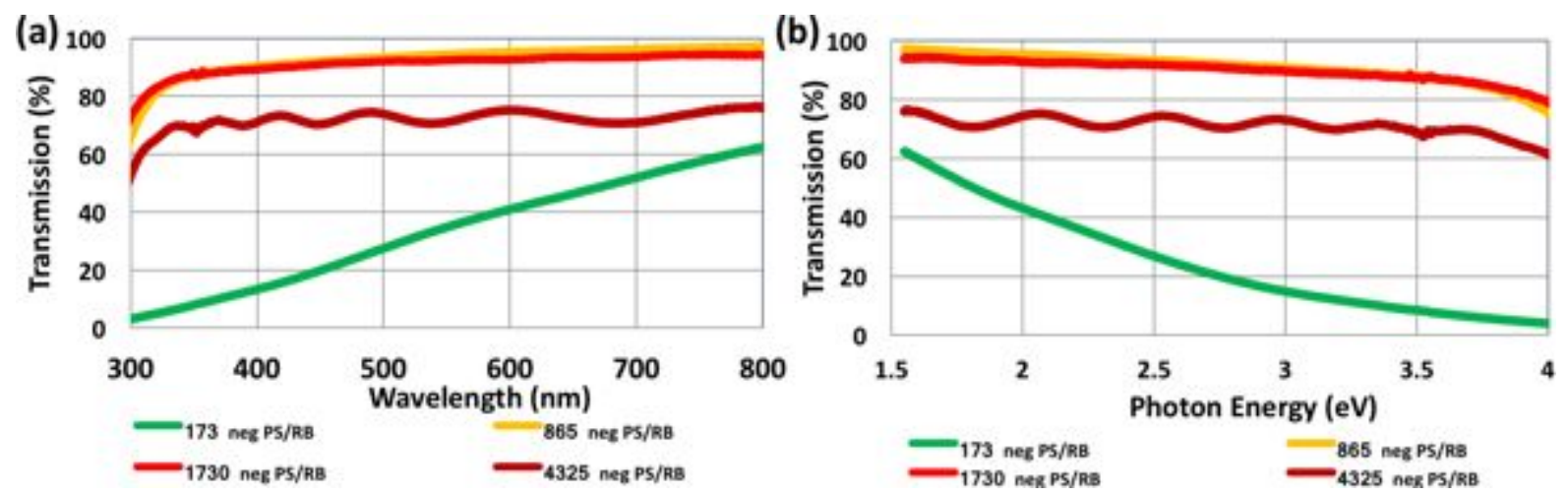

Figure S1. Transmission spectra of the solid-films employing raspberry particles (RB) and negatively charged polystyrene particles (neg PS) at various particle number ratios presented against (a) the wavelength and (b) photon energy. These measurements belong to individual measurements. 


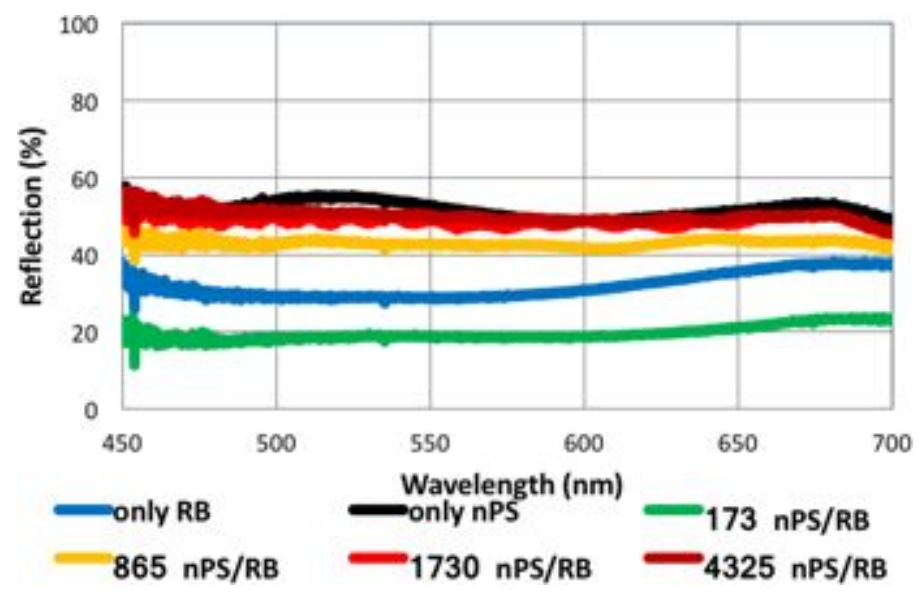

Figure S2. Averaged reflection spectra of the solid-films employing raspberry particles (RB) and negatively charged polystyrene particles (nPS) at various particle number ratios.

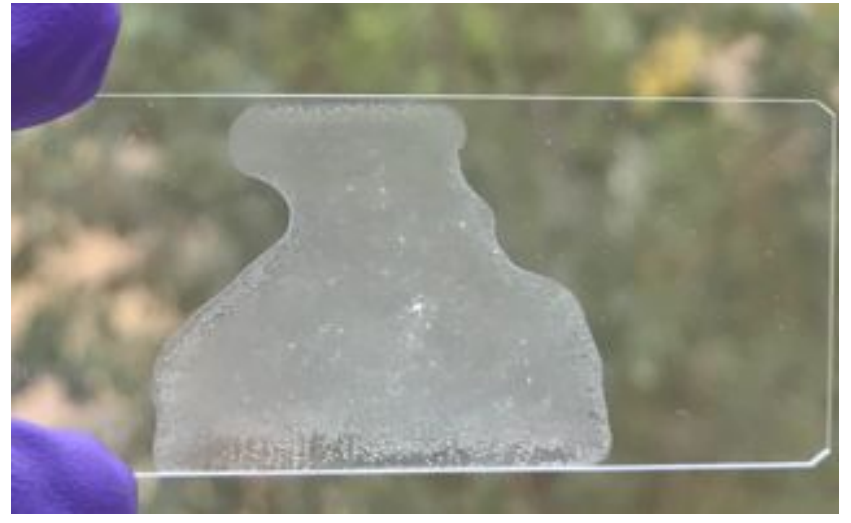

Figure S3. Solid film prepared after centrifuging the raspberry particle and negatively charged polystyrene particle mixture for five times to remove the polystyrene particles that are not attached to raspberry particles as much as possible.

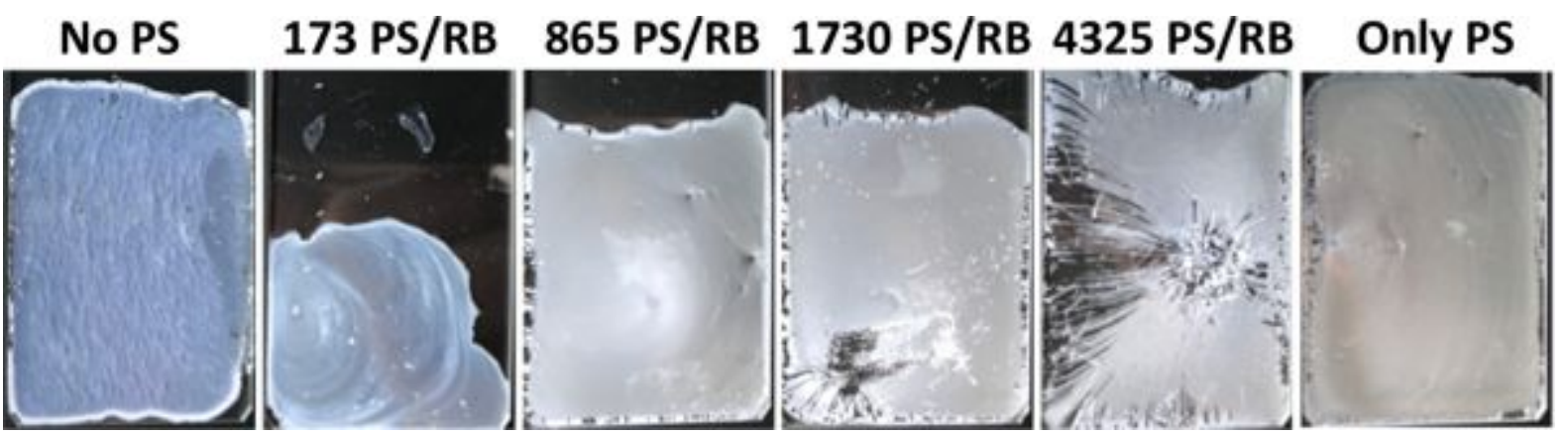

Figure S4. Photographs of the solid films made of only raspberry particles (no positively charged raspberry particle) sample together with hybrid samples with varying positively 
charged polystyrene-to-raspberry particle ratios and only positively charged polystyrene particles.

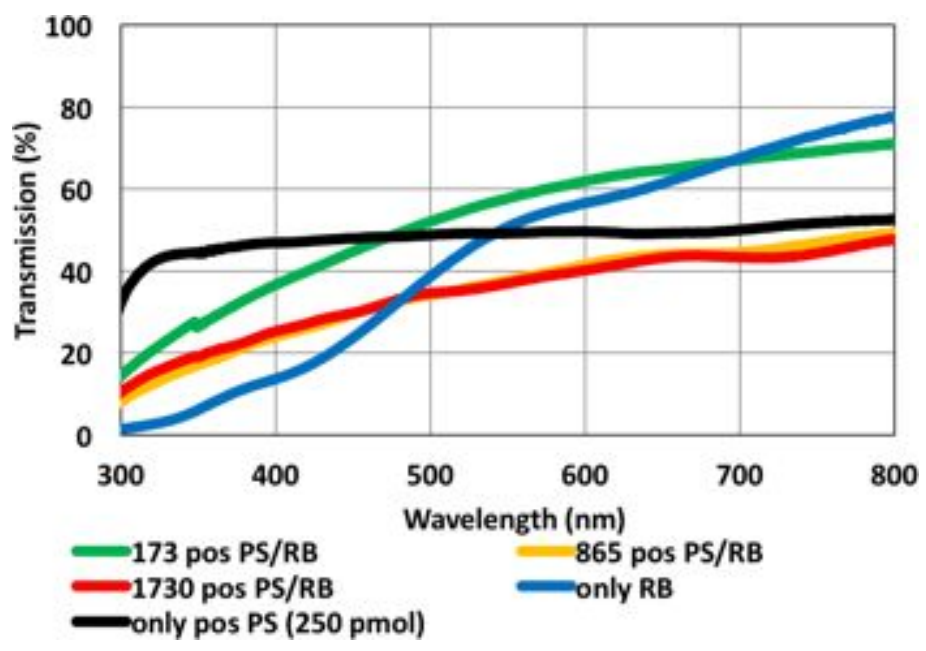

Figure S5. Averaged transmission spectra of the solid-films employing raspberry particles (RB) and positively charged polystyrene particles (pos PS) at various particle number ratios.

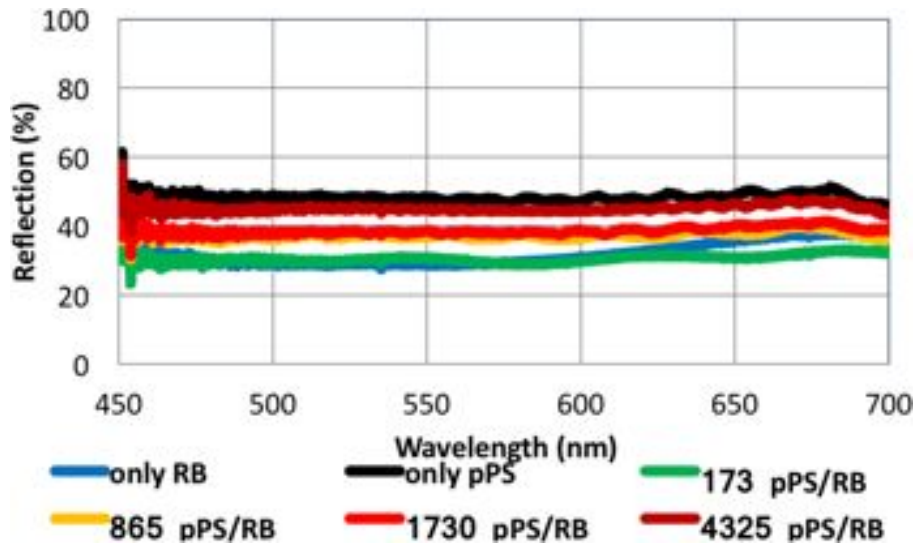

Figure S6. Averaged reflection spectra of the solid-films employing raspberry particles (RB) and positively charged polystyrene particles (pPS) at various particle number ratios. 


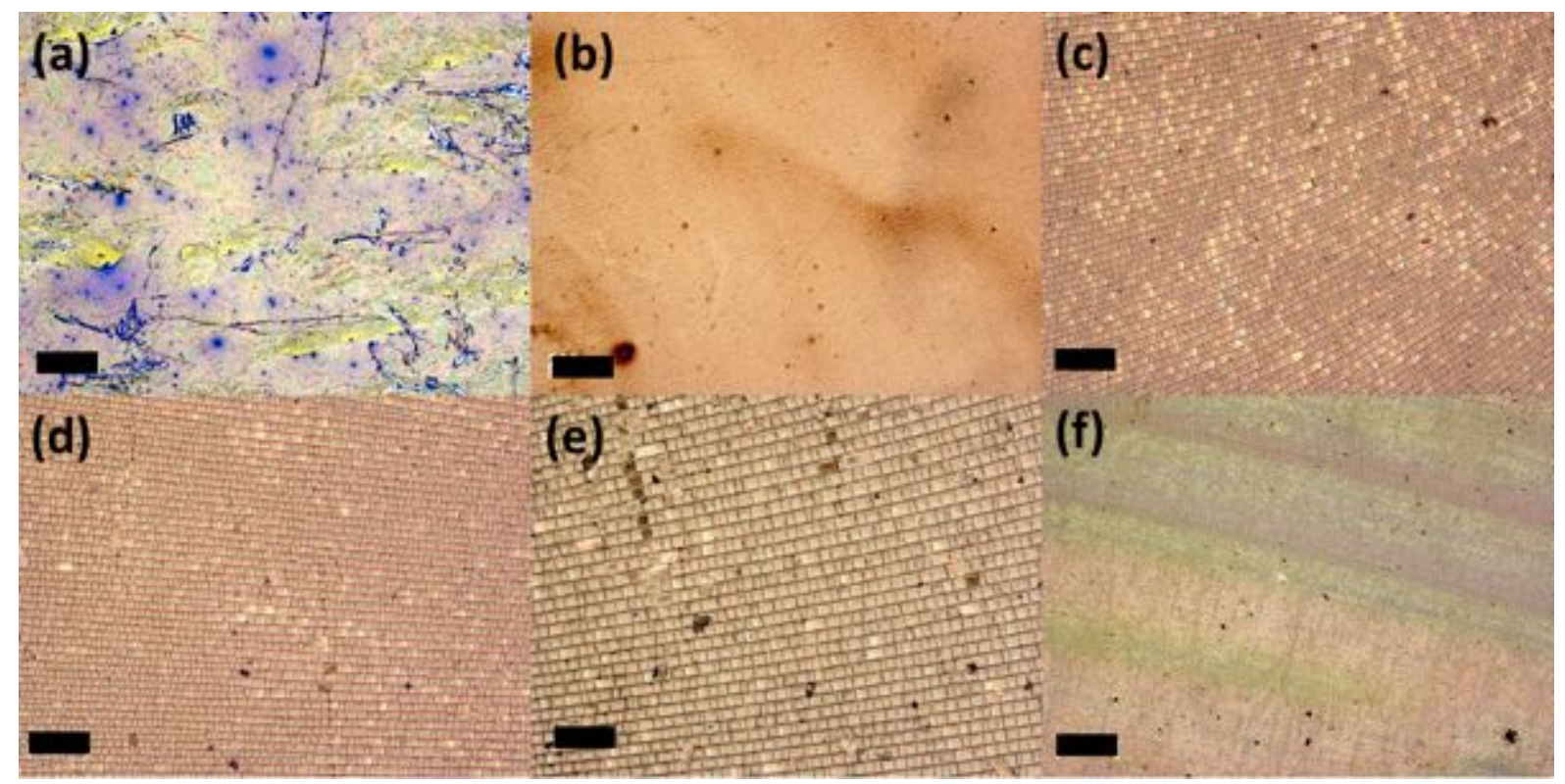

Figure S7. Optical microscopy images of the films made of (a) only raspberry particles along with hybrid samples prepared using (b) 173, (c) 865, (d) 1730 and (e) 4325 positively charged polystyrene particles per raspberry particles, and (f) only polystyrene particle film. Scale bar: $200 \mu \mathrm{m}$.
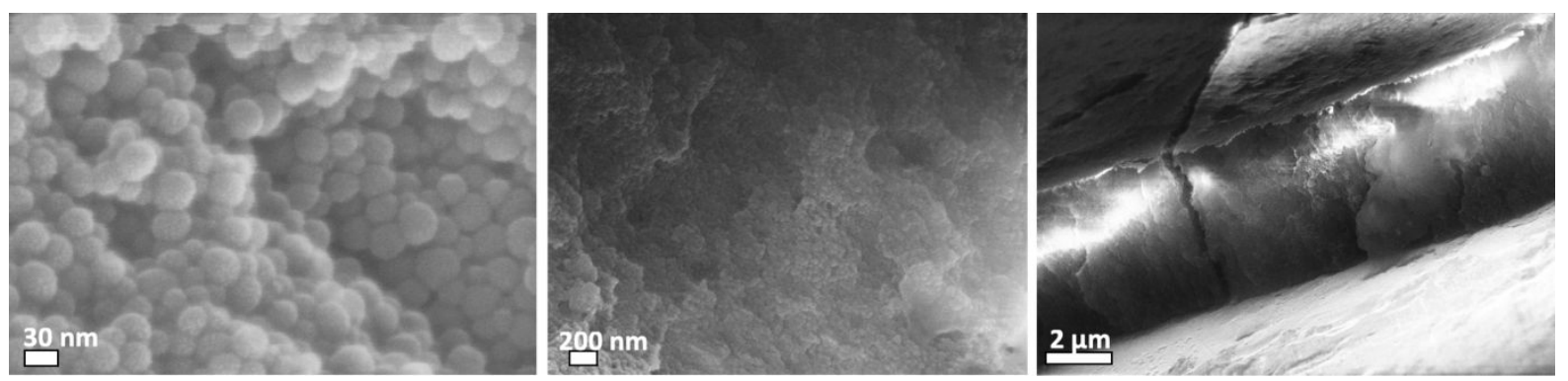

Figure S8. SEM image of the film made of RB particle and positively charged PS particles at various magnifications showing individual PS particles and the structure of the film. 

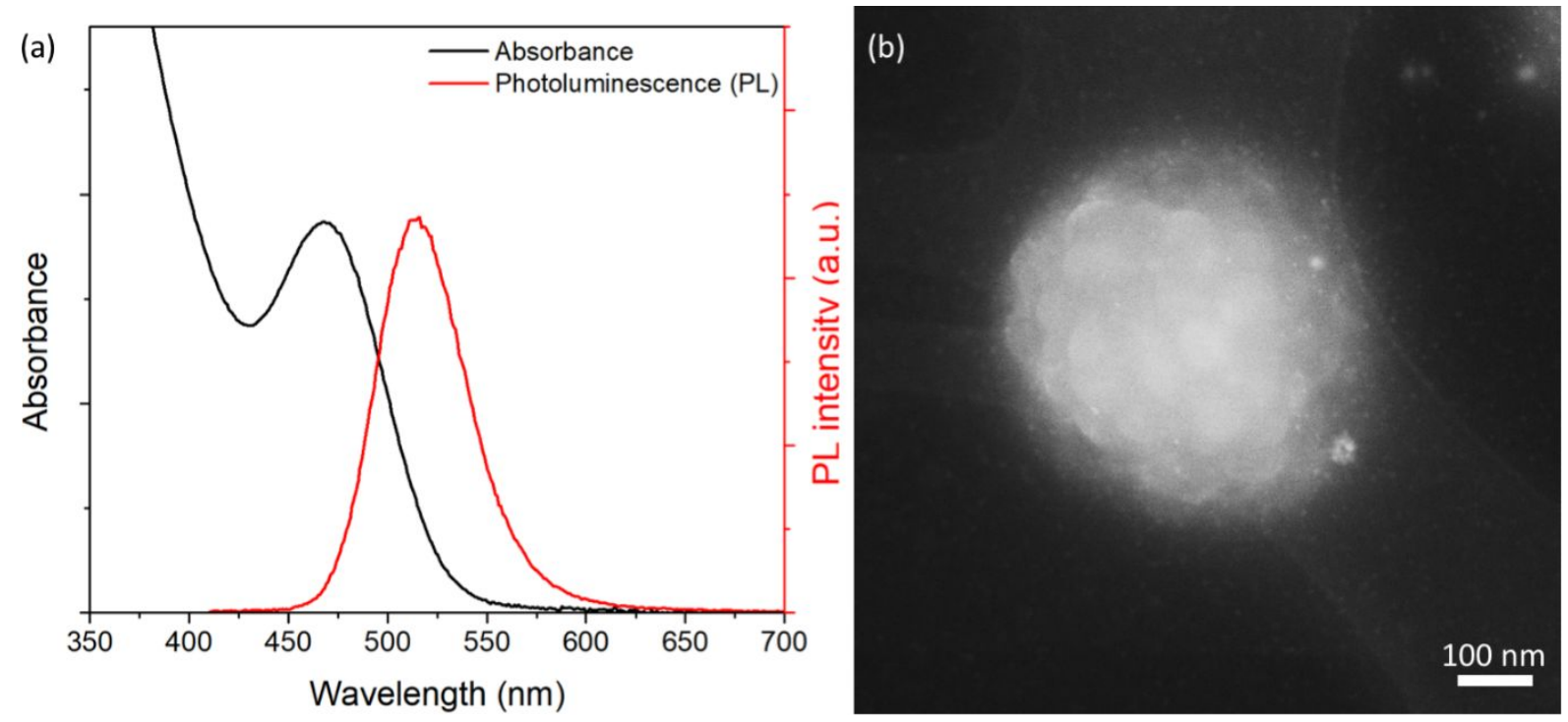

Figure S9. (a) Photoluminescence and absorbance spectra of the quantum dots. (b) HAADF STEM image of raspberry particle and quantum dot hybrid (4325 quantum dot per raspberry particle sample).

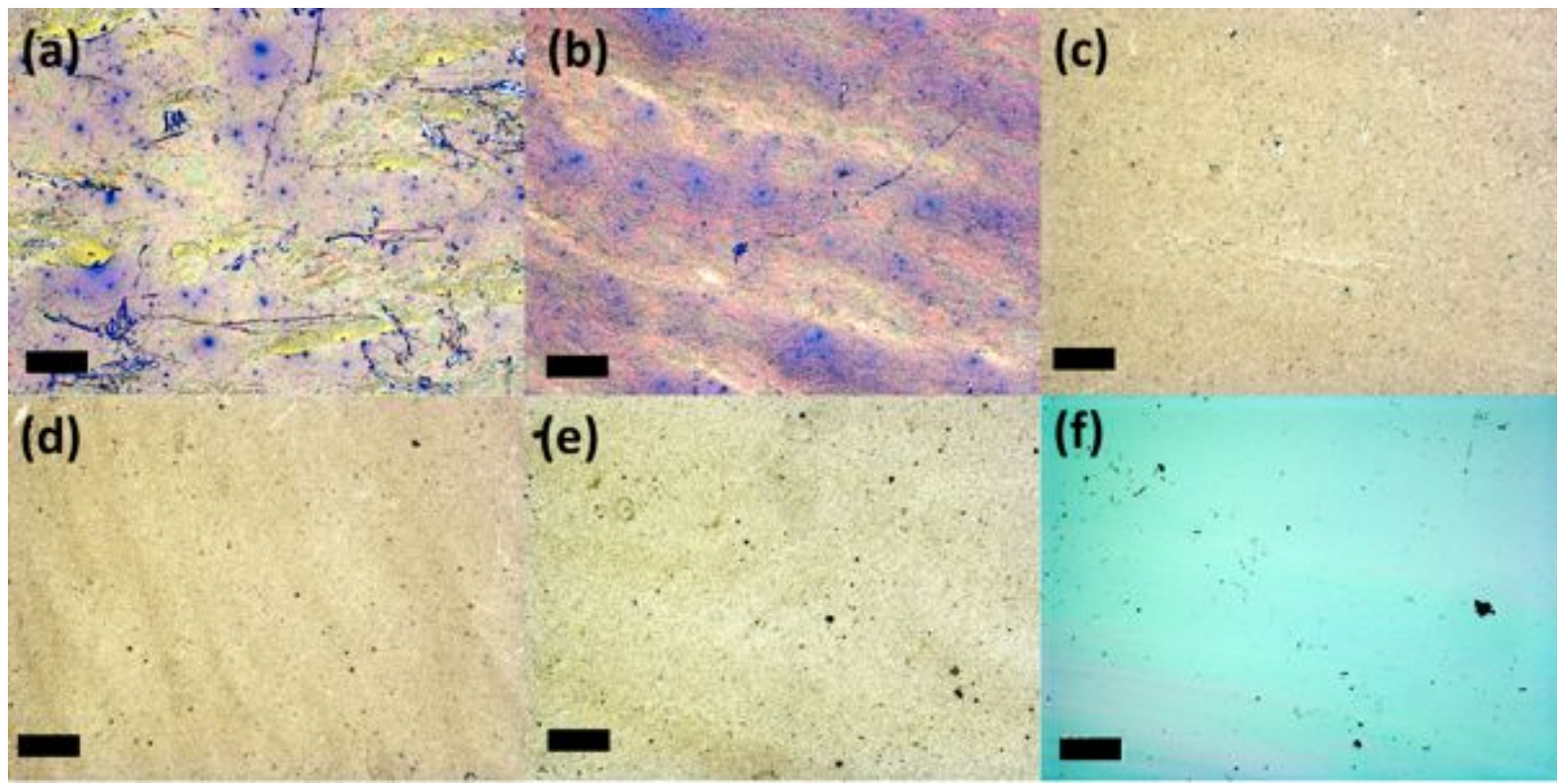

Figure S10. Optical microscopy images of the films made of (a) only raspberry particles along with hybrid samples prepared using (b) 173, (c) 865, (d) 1730 and (e) 4325 quantum dots per raspberry particles, and (f) only quantum dot film. Scale bar: $200 \mu \mathrm{m}$. 


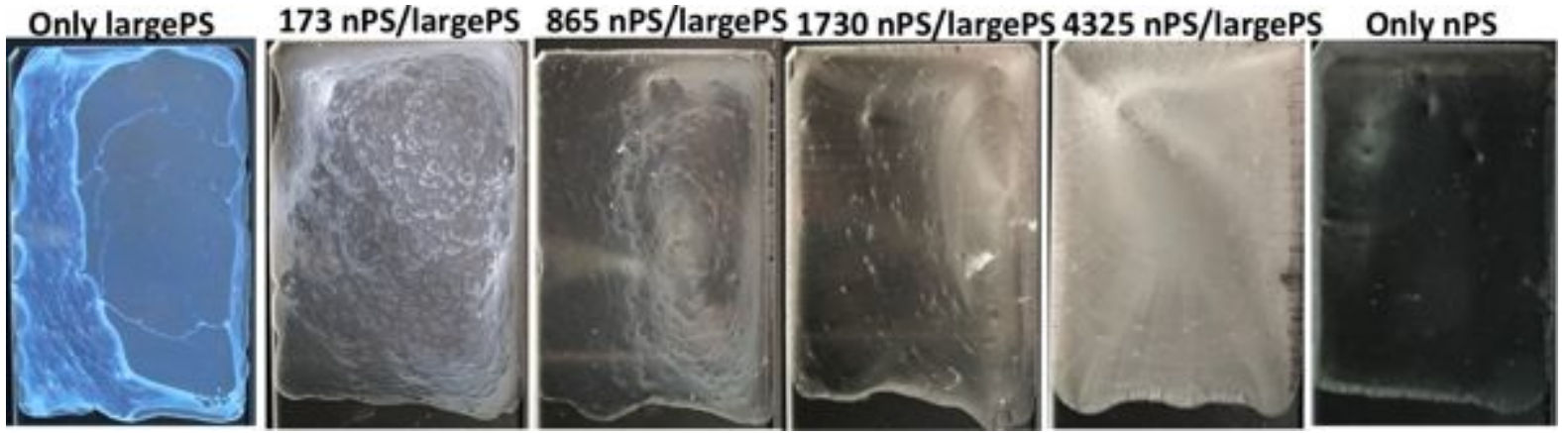

Figure S11. Photographs of the solid films made of only large positively charged polystyrene particles sample together with hybrid samples with varying large positively charged and small negatively charged polystyrene particle ratios and only negatively charged small polystyrene particles.

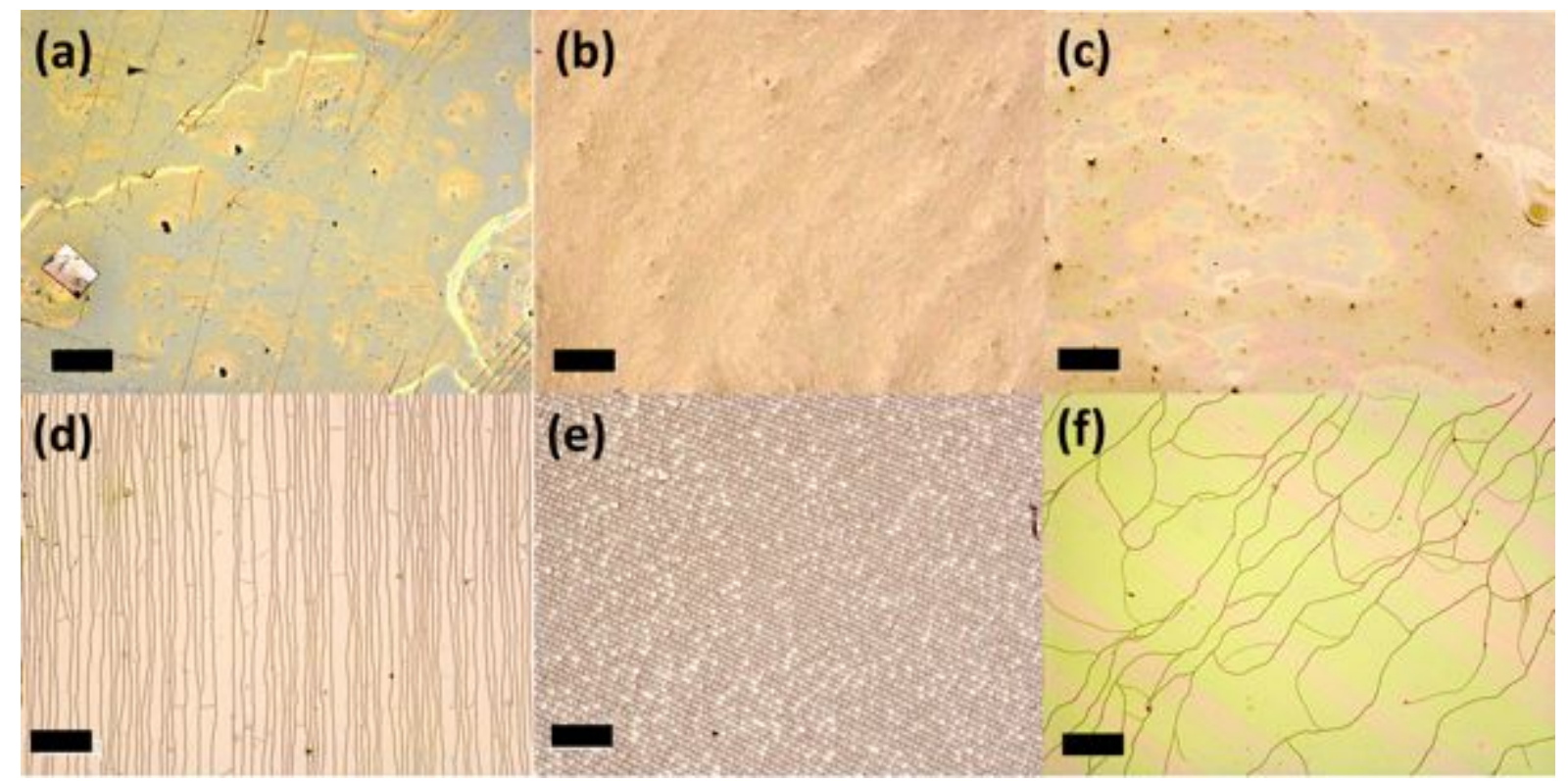

Figure S12. Optical microscopy images of the films made of (a) only large positively charged polystyrene particles along with hybrid samples prepared using (b) 173, (c) 865, (d) 1730 and (e) 4325 negatively charged small polystyrene per positively charged large particles, and (f) only negatively charged polystyrene particles. Scale bar: $200 \mu \mathrm{m}$. 


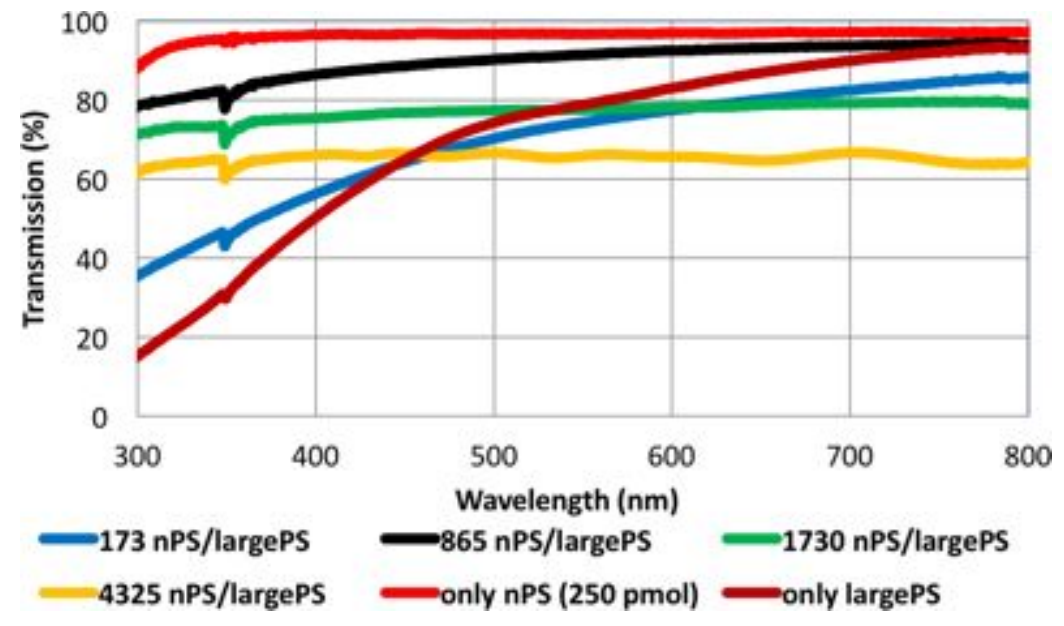

Figure S13. Averaged transmission spectra of the solid-films employing positively charged large polystyrene particles (largePS) and negatively charged polystyrene particles (nPS) at various particle number ratios. 